\section{Dial 'P' for pain}

There is a wealth of evidence to indicate that substance $\mathrm{P}$ (SP), a peptide neurotransmitter that acts at the neurokinin 1 (NK1) receptor, is involved in the perception of pain (nociception). The analgesic action of opioid therapeutics, such as morphine, seems to be explained in part by their ability to decrease SP release, and SP levels are increased in animal models of chronic pain. But despite a clear role for SP in mediating pain states, NK1receptor antagonists have failed to show effectiveness as analgesics in clinical trials. Now, a study that examines the balance between nociceptive transmitter systems in mice provides a hint that NK1-receptor antagonists might be more effective if used as adjuncts to opioid-based analgesia.

A number of neurotransmitters seem to act in concert to control nociception. Under normal conditions, tonic stimulation of $\alpha_{2}$-adrenoceptors by noradrenaline (NA) provides an inhibitory influence, increasing the threshold for nociception and potentiating the analgesic effects of morphine. In the present study, the authors investigated nociceptive behaviour in the absence of NA by studying mutant mice that lacked the gene for the enzyme that is responsible for synthesizing NA, dopamine $\beta$-hydroxylase. An elegant feature of this model was that normal NA levels could be restored by injecting the mutant mice with a drug that is converted to NA by endogenous enzymatic activity. As expected from the presumed inhibitory role of NA in nociception, the mice that lacked NA had a decreased pain threshold, although unexectedly this 'hyperalgesia' was limited to thermal stimuli, but not to mechanically induced pain. Restoring NA levels normalized nociception, showing that the hyperalgesia was specifically due to the lack of NA, not to any developmental abnormality in the genetically altered mice.

In support of the hypothesis that NA exerts its inhibitory effect by reducing levels of SP release, the authors found that the mice that lacked NA had increased levels of SP immunoreactivity in key regions involved in pain transmission. Furthermore, NK1-receptor antagonists were shown to be analgesic in the mutant mice, but not in control mice with normal NA levels, implying that the extent of SP-mediated nociception was greater in mice in the hyperalgesic state. Interestingly, NK1-receptor antagonists were also able to reverse the reduction in the analgesic potency of morphine that was observed in the mutant mice, indicating that morphine cannot completely abolish SP release in the absence of NA.

Dysfunction of the NA-mediated inhibitory pain system therefore seems to produce a specific type of chronic hyperalgesia in which the balance of the opposite actions that SP and opioids have on pain behaviour is altered, and in which SP has a crucial role. This indicates that NK1-receptor antagonists, although not effective when used alone, might prove useful in cases of hyperalgesia that are characterized by a reduced sensitivity to morphine. These findings could pave the way towards pain treatment with less addictive potential than current morphine use.

Adam Smith

Editor, Nature Reviews Drug Discovery

\section{(2) References and links} ORIGINAL RESEARCH PAPER Jasmin, L. et al. The NK1 receptor mediates both the hyperalgesia and the resistance to morphine in mice lacking noradrenaline. Proc. Natt Acad. Sci. USA 99, 1029-1034 (2002)

WEB SITE

Jasmin \& Ohara's lab:

http://anatomy.ucsf.edu/ohara/index.html

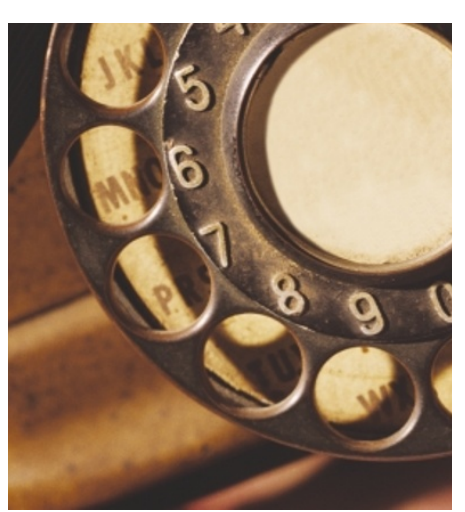

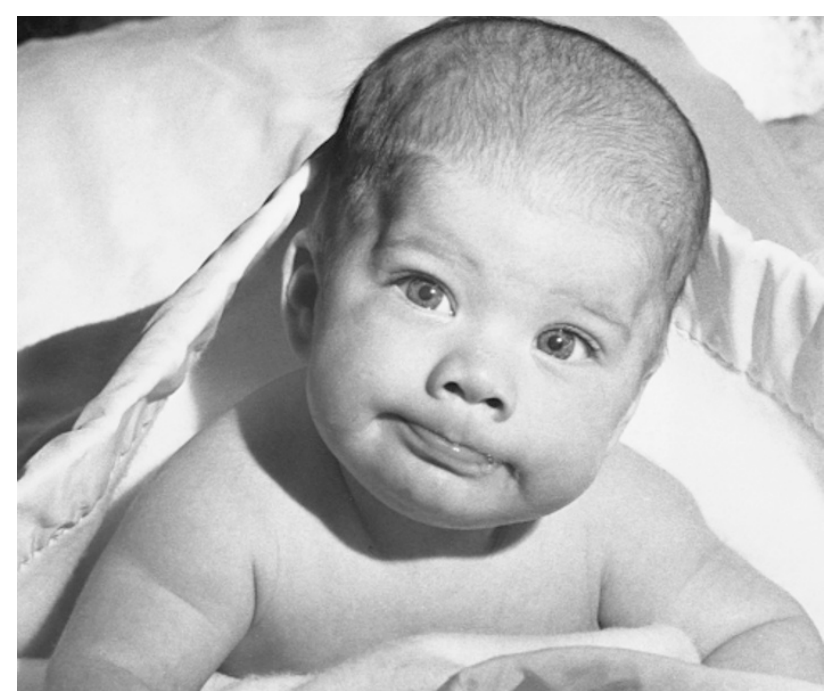

ION CHANNELS

\section{A rebellious last-born}

NMDA ( $N$-methyl-D-aspartate) receptors are heteromeric, $\mathrm{Ca}^{2+}$ permeable channels that require two agonists for their activation - glutamate and glycine. Glycine binds to the so-called NR1 subunit, whereas glutamate binds to any of four different NR2 subunits - NR2A-D. In 1995, a new subunit, NR3A, was independently cloned by Ciabarra et al. and Lipton and colleagues. The function of NR3A is not fully clear, although we know that its co-expression with other NMDA receptor subunits modifies the channel properties. Now Lipton and colleagues have identified NR3B, a new NR3 subunit that, judging by the human genome, is probably the final member of the NMDA receptor family. NR3B sheds some light on the function of the NR3 subunits, but opens just as many intriguing questions.

The main peculiarity of NR3B is that it can form heteromers with NR1, giving rise to cation-selective channels that need only glycine for their activation. Although glycine has its own set of receptors, they are selective for anions and have pharmacological properties that are quite different from the NR3B-containing heteromers. In fact, the pharmacology of NR3B is also atypical in the context of NMDA receptors. So, NR3B is unresponsive to glutamate agonists and antagonists and, although it recognizes NR1-selective glycine agonists, these drugs behave as NR3B antagonists. Moreover, NR3B-containing receptors are not permeable to $\mathrm{Ca}^{2+}$ and show little of the $\mathrm{Mg}^{2+}$-dependent blockade that is characteristic of NMDA receptors. Last, the authors revisited the properties of the previously identified NR3 subunit and found that it could also form glutamate-insensitive, glycine-gated channels when co-expressed with NR1.

What is the functional significance of NR3B in vivo? As a first step, the authors found that glycine can evoke action potentials in cultured neurons, an effect that shared, to some degree, the pharmacological profile of the NR1/NR3A heteromers. But as NR3B is expressed in ventral horn motor neurons, it will be interesting to test directly whether it participates in transmission in the spinal cord, a region in which glycine has long been known to have synaptic actions.

Juan Carlos López

(2) References and links

ORIGINAL RESEARCH PAPER Chatterton, J. E. et al. Excitatory glycine receptors containing ORIGINAL RESEARCH PAPER Chatterton, J. E. et al. Excitatory glycine receptors containing
the NR3 family of NMDA receptor subunits. Nature 30 January 2002 (10.1038/nature715) FURTHER READING Ciabarra, A. M. et al. Cloning and characterization of chi-1: a developmentally regulated member of a novel class of the ionotropic glutamate receptor family. J. Neurosci. 15, 6498-6508 (1995) | Sucher, N. J. et al. Developmental and regional expression pattern of a novel NMDA receptor-like subunit (NMDAR-L) in the rodent brain. J. Neurosci. 15, 6509-6520 (1995) | Cull-Candy, S. et al. NMDA receptor subunits: diversity, J. Neurosci. 15, 6509-6520 (1995) | Cull-Candy, S. et al. NMDA recep
development and disease. Curr. Opin. Neurobiol. 11, 327-335 (2001) 